\title{
BIO-MEDICAL APPLICATIONS OF SYNCHROTRON X-RAY FLUORESCENCE
}

\author{
W.M. KWIATEK \\ H. Niewodniczański Institute of Nuclear Physics \\ Radzikowskiego 152, 31-342 Kraków, Poland
}

\begin{abstract}
Since the exact role of trace elements in living organisms is still not very well known, it is a need of a careful analysis and study on trace element distributions in various bio-medical materials. The conventional techniques such as X-ray fluorescence, proton-induced X-ray emission, atomic absorption spectrophotometry do not always give precise determination, especially in microstructures areas. Since synchrotron radiation is very powerful and gives the opportunity to work with micro-beams the synchrotron radiation induced X-ray emission technique has been recently developed. Due to many properties of synchrotron radiation it becomes a very useful tool in science. In this paper a characterization of different apparatus for a trace element analysis as well as several applications are described to show the usefulness of the synchrotron radiation induced X-ray emission.
\end{abstract}

PACS numbers: 07.85.+n, 87.59.-e, 32.30.Rj

\section{Bio-medical needs for a trace element analysis}

A human and animal body consists of different elements. These elements are grouped into matrix elements and trace elements. Essential matrix elements include: $\mathrm{H}, \mathrm{C}, \mathrm{N}, \mathrm{O}, \mathrm{Na}, \mathrm{Mg}, \mathrm{P}, \mathrm{S}, \mathrm{Cl}, \mathrm{K}$, and $\mathrm{Ca}$. The second group may include all the rest of elements from the periodic table. So far the existence of the following trace elements has been found: $\mathrm{B}, \mathrm{F}, \mathrm{Al}, \mathrm{Si}, \mathrm{V}, \mathrm{Cr}, \mathrm{Mn}, \mathrm{Fe}, \mathrm{Co}, \mathrm{Ni}, \mathrm{Cu}, \mathrm{Zn}, \mathrm{As}$, $\mathrm{Mo}, \mathrm{Sn}, \mathrm{I}$, and even $\mathrm{Hg}$ and $\mathrm{Pb}$.

The trace elements play an important role in living organisms. They act as co-factors in metabolic processes and serve as a component of enzymes [1]. They may serve as inhibitors or promotors of living processes in organisms [2]. One can also say that the trace elements as essential elements for live can be determined as toxic elements. The elemental toxicity is difficult to establish because it depends on a physiological concentration level of the element in the organism. Each increase or decrease in concentration may cause some disease [3].

A study of heavy metals, especially lead and mercury, is very popular in biological science because of their toxic nature. It is very well known that lead 
enters the air, water, and soil as a result of human activities. The major way of lead absorption is inhaling and non-direct consumption. After absorption lead is distributed in soft tissues (kidney and liver) and then redeposited in bone, teeth, and hair [4]. An increased level of lead concentration in a human body may even cause death.

Mercury poisoning has been a problem for many years. Major toxic forms of mercury are: mercury vapor, salts of mercury, and organic mercurials. The central nervous system appears to be the most sensitive to toxic effects of an organic form of mercury. Based on the data presented by Bakir [5] the lethal dose of mercury in blood is $>3 \mu \mathrm{g} / \mathrm{ml}$.

Therefore it is important to perform a trace element analysis study to improve both our knowledge about the role of the trace elements and understanding of the metabolic processes. There are several techniques that can be applied for such an analysis but it seems that one of the most powerful technique is the synchrotron radiation induced X-ray emission (SRIXE) due to synchrotron radiation properties.

It is also important to know the elemental distribution in the organism especially within a selected tissue structure. Such a study will give us information on the structural function as well.

\section{Basic principles of $\mathrm{X}$-ray fluorescence}

The basic principle of X-ray fluorescence is the production of the atomic inner-shell vacancy. Such a vacancy can be produced by bombarding an atom with a particle beam or irradiation it with a photon beam. There are several different phenomena that can occur during the irradiation. One of them is X-ray fluorescence. Since the vacancy has been produced, the emission of characteristic $\mathrm{X}$-rays is possible and then one has to collect and analyze the energy dispersive spectrum. The peak intensity corresponds to the element concentration level. A typical characteristic X-ray spectrum induced by synchrotron radiation is shown in Fig. 1. The detailed description of X-ray fluorescence physics can be found elsewhere [6].

\section{Characteristic of synchrotron radiation properties}

Synchrotron radiation (SR) has many properties that are very useful and make SRIXE very attractive and valuable. These properties include [7]:

- a continuous energy distribution spectrum (a white beam),

- photons are highly polarized in the electron orbit plane,

- photons are highly collimated in the vertical direction,

- a beam is characterized by high brightness, brilliance and flux over a wide range of the energy distribution,

- a beam can be focused. 


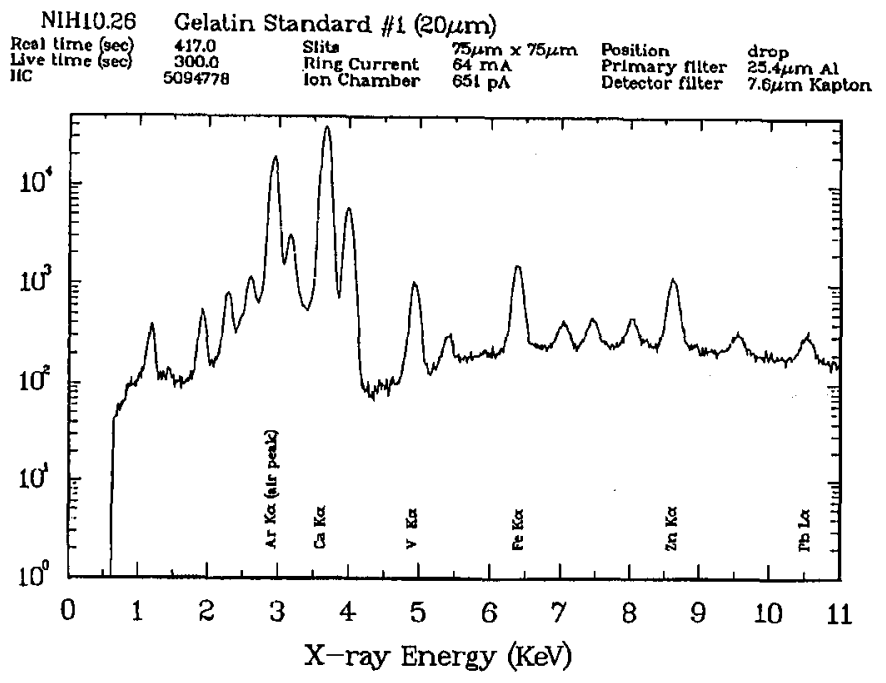

Fig. 1. A typical characteristic X-ray spectrum induced by synchrotron radiation.

These properties resulted in the development of the SRIXE technique for a trace element analysis even on microprobe scale. Since SR produces a white beam it can be monochromatized and still has enough flux for focusing and inducing characteristic X-rays with sufficient statistics.

The elemental analysis can be performed in any environment (air, helium atmosphere). The spectra accumulation time is very short and multi-elemental detection with a very low minimum detectable limit (MDL) is achieved. An analysis can be performed on either a wet or dry sample with a very small amount of it. In addition almost no sample preparation procedure is required before the analysis.

Summarizing the properties of synchrotron radiation resulted in advantages of the SRIXE technique they are as follows:

- multi-elemental detection capability,

- a non-destructive analysis,

- a small sample size,

- good spatial resolution,

- a short analysis time,

- no special requirements for target environment,

- a very low MDL.

\section{Description of experimental apparatus}

Basically there are three set-ups for the trace element analysis with synchrotron radiation:

1. a set-up for the so-called traditional SRIXE - shown in Fig. 2, 
2. a set-up for the total reflection X-ray fluorescence (TRXRF) - shown in Fig. 3,

3. a set-up for micro-SRIXE - shown in Fig. 4.
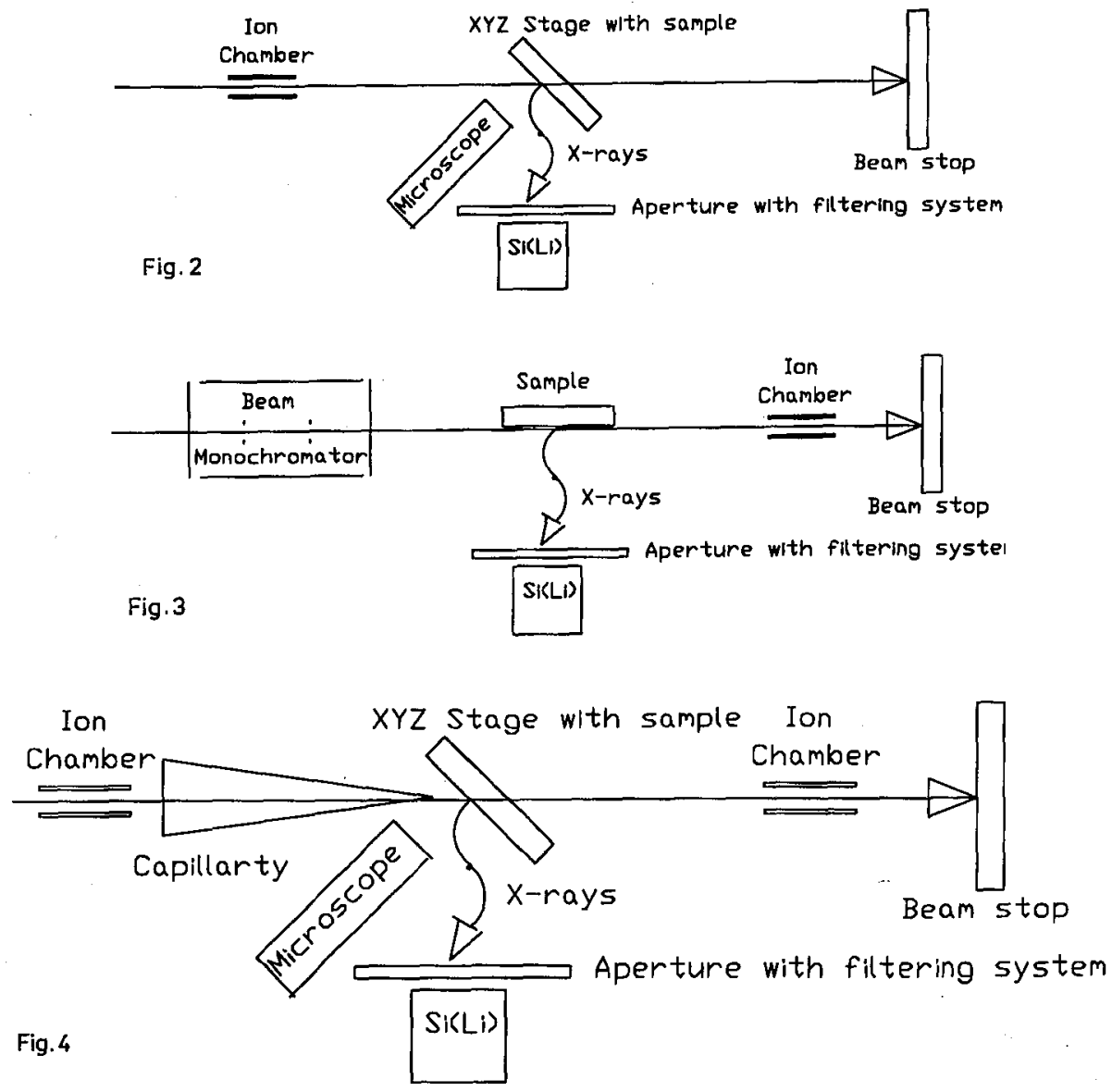

Fig. 2. Set-up for the so-called traditional SRIXE.

Fig. 3. Set-up for the total reflection X-ray fluorescence (TRXRF).

Fig. 4. Set-up for micro-SRIXE.

In all these arrangements photons are delivered through the set of Be windows that protect the high vacuum in a storage ring. In the first set-up the white beam can be cut down with slits to the required size and then passes the helium ionization chamber. Such a chamber may serve as a beam position control device and be used for normalization of the analyzed spectra. Then the beam hits the target placed at an angle of $45^{\circ}$ to the beam. A fluorescent spectrum is detected 
o)

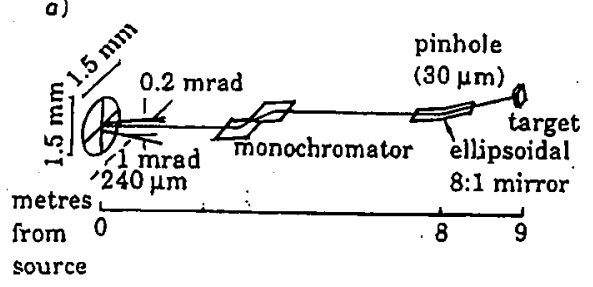

c)

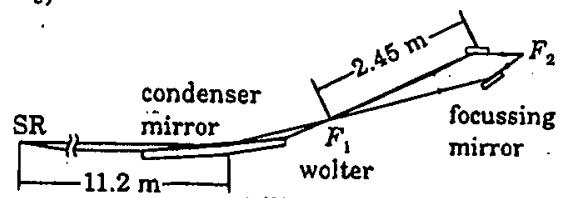

(ellipsoidal thyperbojoidal)

ion

chamber

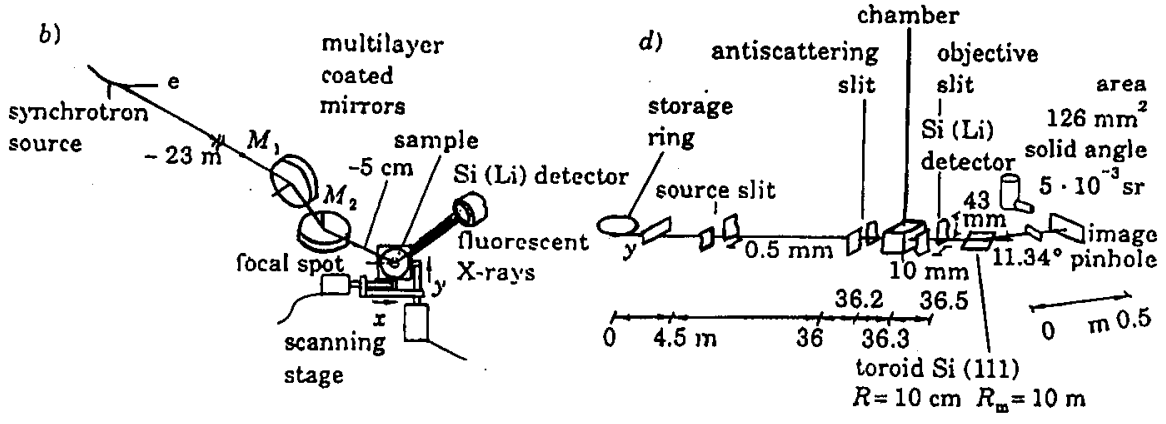

Fig. 5. Different microbeam optics used for a beam focusing.

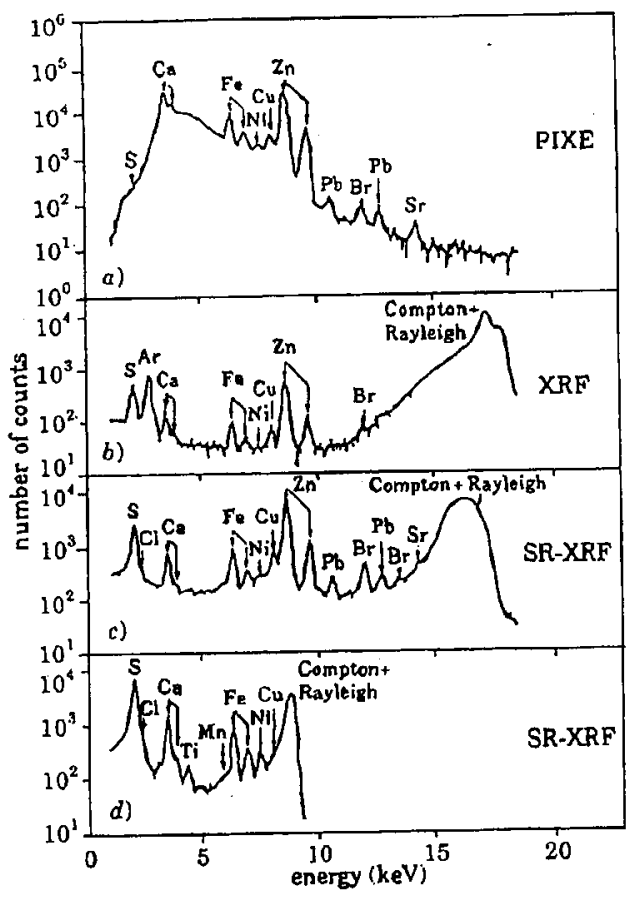

Fig. 6. Human hair spectra taken with different SRIXE set-ups in comparison to conventional $\mathrm{X}$-ray tube and proton induced X-ray emission technique. 
with a $\mathrm{Si}(\mathrm{Li})$ detector positioned in the electron orbit plane at $90^{\circ}$ to the beam in order to minimize the background in the spectrum due to scattered X-rays. In front of the target a TV camera is usually placed in order to view and recognize the beam spot on the target during the measurements. More details about such experimental set-up put on X26 beam line at the NSLS can be found in Ref. [8]. It should be mentioned that with such a traditional SRIXE set-up one can use different types of monochromators to provide measurements with selected photon energy.

The following set-ups put into operation at DORIS storage ring in Hamburg are described in Ref. [9]. The TRXRF technique is devoted to a uniform type of samples that can be prepared in a form of thin target layers placed on a flat silicon crystal. Mainly liquid samples can be analyzed. The set-up with a capillary system allows one to squeeze the photon beam down to $0.1 \mu \mathrm{m}$ in diameter [9].

The other microbeam optics used for a beam focusing are shown in Fig. 5.

The strong requirements for a proper alignment procedure have been already published [8]. The MDL strongly depends on this procedure since the peak-to-background ratio is a critical factor. This may be taken as a disadvantage of the use of synchrotron radiation for the trace element analysis.

Figure 6 compares the spectra of human hair taken with different SRIXE set-ups in comparison to conventional X-ray tube and proton induced X-ray emission technique.

\section{Bio-medical applications}

Trace element studies in bio-medical materials cover an analysis of:

- body fluids: blood, erythrocytes, plasma, serum, urine, and sperm;

- organs taken during surgical operations;

- bones;

- hair and nails.

A soft tissue of the organs can be analyzed in a different form; as a tissue section placed on a thin support film or as a pellet made out of homogenized dried material. According to Iyengar et al. [10], Table summarizes the so-called physiological concentrations of selected trace elements (TE) in different human body parts and fluids.

Depending on a sample type and the studying problem there are several sample preparation procedures. These are very well described by Valkovic in IAEA Tech. Doc [11].

The changes of TE concentration levels may indicate some disease if they are correlated with the clinical tests. As an example of elemental changes in normal and cancerous tissue section Fig. 7 shows three different spectra taken at Hasylab [12].

Very often the elemental concentration changes in the organism are caused by a polluted environment. Therefore it seems to be important to analyze environmental samples in order to study the influence of the pollution to health. The 
TABLE

Concentrations of selected TE in human body fluids and tissues in ppm.

\begin{tabular}{c|c|c|c|c|c}
\hline \hline Element & Blood & Bone & Brain & Hair & Kidney \\
\hline $\mathrm{S}$ & 1680 & 500 & 1500 & $4 \%$ & 2100 \\
$\mathrm{Cl}$ & 2880 & 630 & 1400 & 950 & 2300 \\
$\mathrm{~K}$ & 1620 & 1470 & 185 & 150 & 179 \\
$\mathrm{Ca}$ & 60.5 & $27 \%$ & 7.6 & 2100 & 7.4 \\
$\mathrm{Fe}$ & 447 & 40 & 4100 & 44.7 & 88 \\
$\mathrm{Cu}$ & 1.01 & $1-4$ & 22.5 & 19 & 14.2 \\
$\mathrm{Zn}$ & 7.0 & 170 & 56 & 220 & 49 \\
$\mathrm{Br}$ & 4.7 & 40 & $1-50$ & 25 & 6.7 \\
$\mathrm{Rb}$ & 2.4 & 0.1 & $4-18$ & 0.2 & 20 \\
$\mathrm{Sr}$ & 0.03 & 160 & 3.1 & 0.15 & 7.6
\end{tabular}
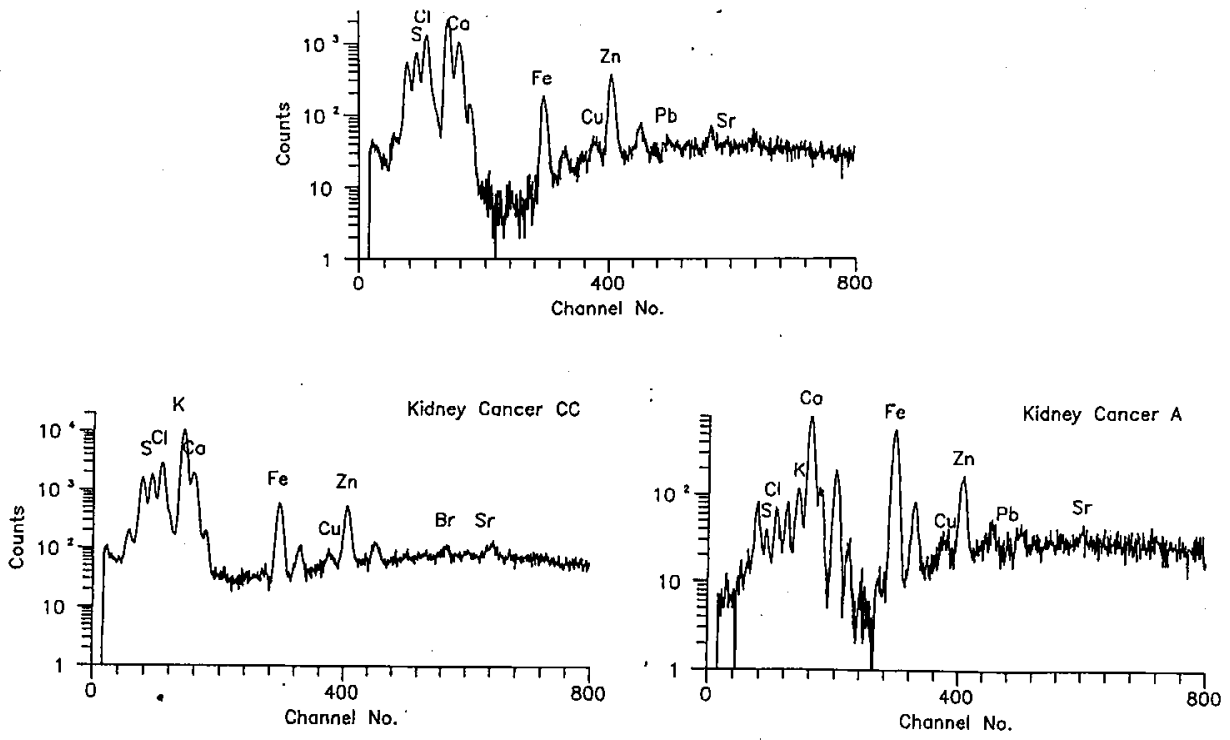

Fig. 7. Elemental changes in normal and cancerous kidney tissue section.

micro SRIXE technique allows one to analyze very small objects as for example fly ashes. It is known that the particles below $10 \mu \mathrm{m}$ are dangerous for humans. The most dangerous are the particles, with a size of less than $2 \mu \mathrm{m}$ in diameter. Figure 8 shows the X-ray intensity maps obtained from the scan over the fly ash particles (after Jaksic et al. [13]). Such a study is important due to better understanding of the combustion process and the influence of the environment to health. 

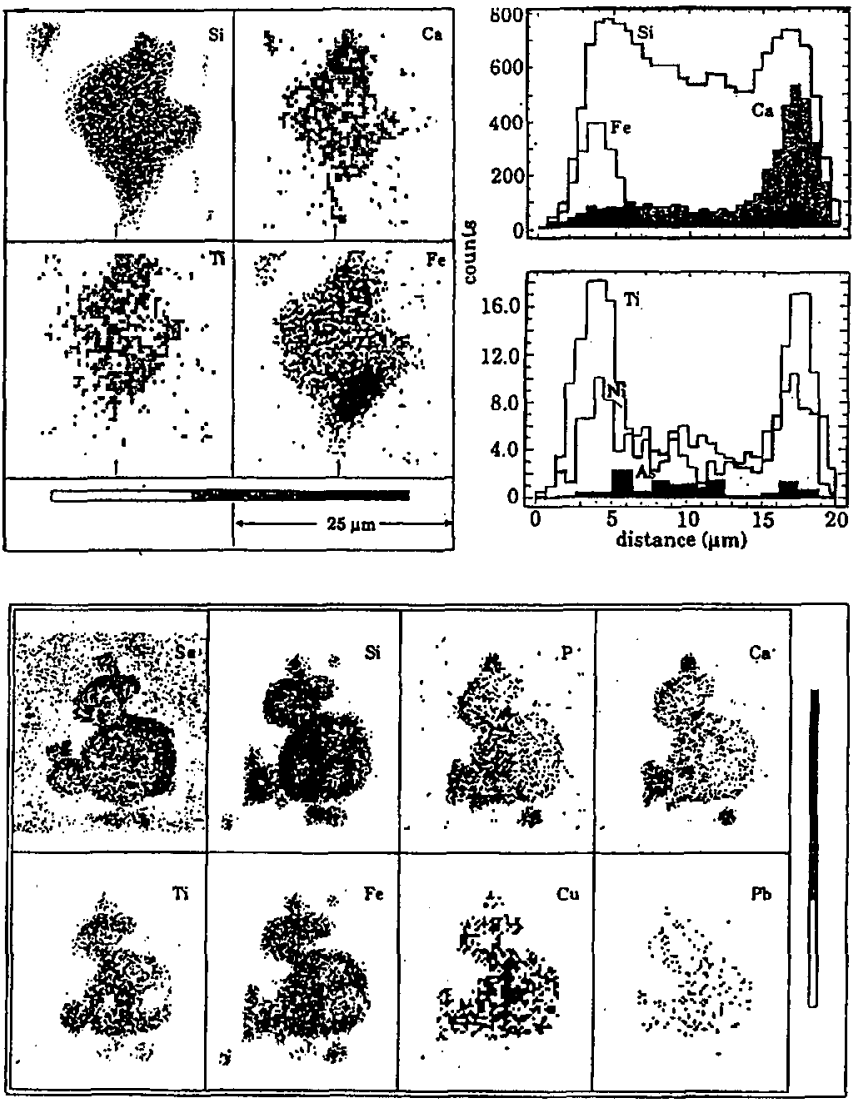

Fig. 8. The X-ray intensity maps obtained from the scan over the fly ash particles [13].

\section{Summary}

The SRIXE technique due to its advantages is a very useful and appropriate technique for bio-medical applications. In addition to the trace element analysis done by SRIXE one should also consider a complementary study such as determination of chemical compounds composition. This can be performed by means of FTIR spectroscopy, EXAFS, or XANES [7]. These techniques can determine the bonds between the elements as well as oxidation states of metals. This type of a simultaneous complementary analysis will be very valuable in the future development of bio-medical applications of synchrotron radiation. Also, possibilities of micro-computed tomography with SR may play an important role in that development and will cause an obvious increase in the interest of SR applications in this field.

Since TE seem to play an important role in living organisms and their concentration level is very low, still, many measurements have to be performed in the future. 


\section{Acknowledgments}

The work presented in this paper was partially performed and supported by both Brookhaven National Laboratory, Upton, NY, USA, and HASYLAB at DESY in Hamburg, Germany. The support from the State Committee for Scientific Research (Republic of Poland) is also acknowledged.

\section{References}

[1] J. Prohaska, Physiological Rev. 67, 858 (1987).

[2] R. Scott, B.W. East, J. Janczyszyn, K. Boddy, A.J. Yates, Urolog. Res. 8, 167 (1980).

[3] Trace Elements in Health, Ed. J. Rose, Butterworth Co. Ltd., London 1983.

[4] M. Murakami, K. Hiroszawa, Nature 245, 153 (1973).

[5] F. Bakir, Bulletin WHO 53, 87 (1976).

[6] W.M. Kwiatek, Acta Phys. Pol. A 82, 263 (1992).

[7] K.W. Jones, in: Handbook of X-Ray Spectrometry, Eds. R. Van Grieken, A. Markowicz, Marcel Dekker Inc., New York 1992, p. 441.

[8] W.M. Kwiatek, A.L. Hanson, K.W. Jones, Nucl. Instrum. Methods Phys. Res. B 50, 347 (1990).

[9] A. Buttkewitz, F. Grabe, G. Gaul, A. Knochel, F. Lechtenberg, P. Engstron, S. Larson, A. Rindby, HASYLAB Progress Report, 577 (1990).

[10] G.V. Iyengar, W.E. Kollmer, H.J.M. Bowen, The Elemental Composition of Human Tissues and Body Fluids, Verlag Chemie, Weinheim 1978.

[11] V. Valkovic, IAEA Tech. Doc 300, Vienna 1990.

[12] W.M. Kwiatek, T. Cichocki, M. Gałka, C. Paluszkiewicz, Nucl. Instrum. Methods Phys. Res. B 68, 122 (1992).

[13] F. Jaksic, F. Watt, G.W. Grime, E. Cereda, G.M. Braga Marcazzon, V. Valkovic, Nucl. Instrum. Methods Phys. Res. B 56/57, 699 (1991). 